\section{COLGADOS: EL HURTO DE ENERGÍA ELÉCTRICA COMO RESISTENCIA A LA NOCIÓN DE USUARIO RACIONAL-ECONÓMICO EN CHILE (1980-1986)}

\author{
Mónica Humeres \\ Universidad Diego Portales, Chile \\ http://orcid.org/0000-0002-9555-035X \\ monica.humeres@udp.cl
}

Cómo citar este artículo/Citation: Humeres, Mónica; (2021). Colgados: el hurto de energía eléctrica como resistencia a la noción de usuario racional-económico en Chile (1980-1986). Arbor, 197(801): a617. https://doi.org/10.3989/arbor.2021.801007

Recibido: 14 junio 2020. Aceptado: 7 junio 2021.

Publicado: 9 diciembre 2021.

RESUMEN: Las infraestructuras de la energía se han visto tensionadas por una serie de demandas y responsabilidades que tienden a solucionar problemas cruciales de la actualidad y del futuro, como el cambio climático y la justicia distributiva. En este artículo planteo que, para armonizar muchos de estos asuntos, una mejor comprensión de la relación entre usuarios e infraestructuras debe ser alcanzada. Para ello, sostengo que los estudios infraestructu-rales, en combinación con los análisis de usuarios provenientes de los estudios de Ciencia, Tecnología y Sociedad (CTS), pueden contribuir significativamente a esa tarea. De este modo, desde una perspectiva genealógica y mediante el análisis de documentos de archivo de la Administración Pública de Chile, analizo el caso de los colgados: grupo de usuarios de la energía eléctrica sin re-lación contractual con las empresas de distribución, que emergió como un problema durante la década de los ochenta, cuando se comenzó a privatizar la infraestructura en medio de la dictadura militar. El análisis del caso de los colgados permite notar que, a pe-sar de la evidencia presentada en los estudios encargados para el conocimiento del perfil de este tipo de usuarios, quienes estaban a cargo de la infraestructura empresarios, economistas y políti-cos- interpretaron en todo momento los resultados de dichos es-tudios dentro del marco racional-económico con el que se diseñan las políticas públicas a partir de esa época en Chile. Así, este caso de estudio sirve como un ejemplo de la obstinación con que se aplican los supuestos de racionalidad-económica. A pesar de la evi-dente contradicción entre el comportamiento de los usuarios y los supuestos con que operaban las políticas públicas, la ilusión de la racionalidadeconómica como explicación representativa del comportamiento de los usuarios, continuó siendo el eje articulador de las relaciones infraestructurales.

PALABRAS CLAVE: usuarios; infraestructura; sociología de la energía; políticas sociotécnicas; racionalidad-económica.
Copyright: (c) 2021 CSIC. Este es un artículo de acceso abierto distribuido bajo los términos de la licencia de uso y distribución Creative Commons Reconocimiento 4.0 Internacional (CC BY 4.0).

ABSTRACT: Energy infrastructures have been under pressure by a series of demands and responsibilities to solve crucial problems, both present and future, such as climate change and distributive justice. In this article I argue that, to harmonise many of these issues, a better understanding must be reached of the relationship between users and infrastructure. To this end, I maintain that infrastructure studies, in combination with user analyses from Social Studies of Science, Technology and Society (STS), can significantly contribute to this task. In this way, from a genealogical perspective and through the analysis of archival documents from the Public Administration of Chile, I analyse the case of the colgados: a group of electrical energy users without a contractual relationship with the distribution companies, which emerged as a problem during the 1980 s when the electrical infrastructure began to be privatised during the military dictatorship. Analysis of the case of the colgados shows that, despite evidence presented in studies commissioned to learn about the profile of this group of users, those in charge of the infrastructure at the time - businessmen, economists, and politicians - interpreted the results of these studies within the economic-rational framework with which public policies were designed as of that time in Chile. Thus, this case study serves as an example of the obduracy with which assumptions of economic-rationality are applied. Strikingly, despite the evident contradiction between user behaviour and the assumptions under which public policies operated, the illusion of economicrationality as the most representative explanation of user behaviour continues to be the central focus of infrastructural relationships in the present, whose most common device is the electricity tariff.

KEYWORDS: users; infrastructure; sociology of energy; sociotechnical policies; economic-rationality. 


\section{INTRODUCCIÓN}

En las ciencias sociales recientemente se ha comenzado a hablar de «momento infraestructural» de la energía eléctrica, para dar cuenta de una atención creciente hacia estas entidades como objeto de estudio. Esto ha sucedido debido a los asuntos sociales y políticos que los rodean a nivel internacional (Castán-Broto, 2017), tales como las demandas por justicia social, equidad o el cuidado del medio ambiente. La atención a las infraestructuras, asimismo, ha dejado ver que estos entramados son relevantes pues, a través de ellos se organiza la vida cotidiana individual y conecta también nuestra experiencia ciudadana en la convivencia nacional. Pero más allá de la energía, el análisis de las infraestructuras reviste un campo de convergencia analítica que recientemente se ha enriquecido desde diversas perspectivas, por ejemplo, en relación con el espacio urbano (Graham y Marvin, 2001; Farías, 2011), donde destaca también la mirada histórica a partir de estudios latinoamericanos (Venkatesan, et al., 2018; Harvey y Knox, 2015). Esto no es casual, pues, si en la actualidad las infraestructuras experimentan un momento crítico, esto sucede también como consecuencia de su trayectoria histórica. En el caso específico de las infraestructuras de servicios básicos, como la eléctrica, se ha planteado un debilitamiento general de su universalidad $y$, en muchos lugares, su financiarización ha impuesto más de una contradicción (Venkatesan, et al., 2018).

Una de las entidades más relevantes de las infraestructuras son sus usuarios; sin ellos, estas carecerían de sentido. Por ello, en este artículo sitúo la mirada sobre cómo han sido entendidos y administrados los usuarios de la infraestructura eléctrica en Chile. Tal como lo expresa Tomás Sánchez-Criado (2016), las infraestructuras nos plantean propuestas de vida concretas y, en ese sentido, constituyen una invitación hacia un estilo de vida particular. Pero esa invitación no siempre es universal u homogénea, no involucra del mismo modo a todos los ciudadanos de una misma sociedad.

Así, analizo la emergencia del caso que en Chile se denominó como colgados: usuarios de electricidad que no tienen una relación contractual de clientes con las empresas de distribución y realizan conexiones autogestionadas con la red eléctrica. En la década de los ochenta en este país extremo del cono sur, los intentos por gobernar a los colgados reflejan un choque frontal de la visión que se estaba imponiendo en medio de la dictadura militar sobre los servicios de infraestructuras básicas. De este modo, sostengo que la imposibilidad de los expertos de comprender el comportamiento de los usuarios hizo que fracasaran muchas políticas públicas privatizadoras que pretendían gobernarlos.

De esta manera, esta investigación socio-histórica cuestiona las políticas y a los expertos del periodo de la privatización de la infraestructura eléctrica chilena en su esfuerzo por gobernar ciudadanos/usuarios (clientes o no). Considerando los estudios infraestructurales, así como los de usuarios desde una perspectiva de los estudios de Ciencia, Tecnología y Sociedad, este artículo está orientado a responder a las siguientes interrogantes: ¿De qué manera las políticas infraestructurales comprendieron y administraron a los usuarios en el período de privatización? ¿Qué papel jugó el modo en que estas políticas definieron, y así inscribieron, a los usuarios en la infraestructura eléctrica? ¿Quiénes resistieron la versión de usuario proyectada desde las políticas infraestructurales? Para responder, se analizaron fuentes primarias correspondientes a documentos del Archivo Nacional de la Administración (ARNAD), principalmente los fondos de la Comisión Nacional de Energía (CNE), los de la Empresa Nacional de Electricidad (ENDESA), el Fondo Chilectra y los de la Oficina de Planificación Nacional (ODEPLAN).

\section{INFRAESTRUCTURAS Y USUARIOS: PERSPECTI- VAS TEÓRICAS PARA UN ANÁLISIS SOCIOLÓGICO}

\subsection{El usuario en tiempos de privatización: investiga- ciones sobre el período de reformas neoliberales en Latinoamérica y en Chile}

En los primeros años de los estudios infraestructurales, las infraestructuras fueron conceptualizadas como entidades invisibles (Star y Ruhleder, 1995). Las académicas que enfatizaron este punto de vista, lo hicieron con el objetivo de hacer énfasis en la normalización de la presencia de estas entidades. Es decir, de este modo, se subrayó el hecho de que los usuarios damos por garantizada la existencia y el correcto funcionamiento de las infraestructuras, hasta un punto en que obviamos su existencia. Este atributo de invisibilidad, sin embargo, ha sido cuestionado por quienes estudian infraestructuras latinoamericanas (Hetherington y Campbell, 2014; Velho y Ureta, 2019; Venkatesan et al., 2018; Collier, Mizes, y von Schnitzler, 2016), para hacer hincapié en las características propias de la región. En estos países, las infraestructuras marcaron fuertemente el 
poder colonial, luego el poder del Estado, precisamente, como una manera de hacer visible la intervención humana sobre áreas consideradas demasiado naturales, y el camino -sin fin- hacia el desarrollo. En este sentido las infraestructuras fueron muchas veces emplazadas como signos de sí, construidas para difundir una suerte de conciencia nacional (Bridge, Özkaynak y Turhan, 2018).

De este modo, en Latinoamérica la electrificación se fue propagando de manera estrechamente vinculada a los Estados junto a la promesa del desarrollo y bajo el ideal industrial: los trabajadores producirían más, en menor cantidad de tiempo, aumentarían los indicadores de producción nacional que, a su vez, eran interpretados como el reflejo de un mejor estándar de vida. Sin embargo, la misión y carácter de las infraestructuras en Latinoamérica cambió durante la década de los ochenta. El giro hacia la política neoliberal, las presiones del Fondo Monetario Internacional (Hetherington y Campbell, 2014), y el uso del ideal de la eficiencia como una manera de reducir la propiedad estatal propiedad estatal, pusieron en el horizonte al crecimiento económico como objetivo del desarrollo infraestructural. De esta forma, las infraestructuras se reconvirtieron en objetos financieros, privatizados, descentralizados y en progresiva internacionalización (Venkatesan, et al., 2018).

En este sentido, podría decirse que las infraestructuras latinoamericanas sufrieron una integración subordinada (Varsavsky, 1969; Kaltenbrunner y Painceira, 2018); es decir, una integración al mercado financiero internacional desde la periferia tanto geográfica como simbólica: una posición desde la que se actúa según "los intereses sociales, cognitivos y económicos de los grupos e instituciones centrales» (Kreimer, 2006: 206). En el ámbito infraestructural, y tal como apunta Pirez (2013) «se da por la subordinación de la acumulación de capital, y la transferencia de buena parte del excedente a las economías centrales, frenando el desarrollo de las fuerzas productivas y limitando el dinamismo local» (Pirez, 2013: 48). Asimismo, señala el autor, este tipo de financiarización ha estado caracterizado por una importante exclusión de población, tanto en su condición de (no)consumidores de los servicios básicos, como en su papel de trabajadores (o trabajadores precarios). Todo esto, como consecuencia de la subordinación, por parte de los actores que intervienen en la producción distribución y consumo, de los principios normativos fundamentales a las orientaciones del mercado.
El caso chileno, tiene una doble relevancia en este contexto. Por una parte Chile fue escenario temprano del neoliberalismo (Plehwe, 2009; Dean, 2014; Klein y Fuentes, 2007; Fischer, 2009) y, al mismo tiempo, la industria eléctrica fue una de las primeras en ser privatizadas, proporcionando un ejemplo experimental que fue considerado por muchos otros países de la región (Maldonado y Palma, 2004), y de fuera de ella (Bridge, Özkaynak y Turhan, 2018). Cabe señalar que, en este país del cono sur, las reformas neoliberales y privatizadoras se produjeron además en medio de la dictadura militar (1974-1989) y luego de que la Junta Militar tomara partido por las políticas neoliberales importadas por un grupo de economistas chilenos con postgrados en Chicago (conocidos popularmente como los Chicago Boys) con el objetivo de romper con el diseño socialista instalado por la derrocada Unidad Popular (Lüders, 2012; Arancibia y Barlat, 2007). Así, la estrategia de esta alianza militar-económica implementada por estos expertos fue mucho más allá de deshacer las políticas socialistas: instaló un diseño de sociedad que caló profundo en las instituciones y renovó, según sus propios principios y mecanismos, diversas áreas de la sociedad (Gárate, 2012; Ffrench-Davis, 2003; Undurraga, 2014).

El proceso de privatización del sector eléctrico que se fraguó durante los últimos años de la década de los setenta y comenzó a ser ejecutado a partir de 1980, ha sido estudiado principalmente desde la historia económica y la ingeniería (Estache y Rodriguez-Pradina, 2000; Fisher y Serra, 2007; Maldonado y Palma, 2004). Estos análisis han tendido a evaluar la privatización como proceso y a compararlo dentro de la región latinoamericana, destacando la influencia de Chile sobre la región; en particular sobre Argentina, Bolivia, Perú, Brasil y Colombia (Maldonado y Palma, 2004). En cuanto a las evaluaciones de los resultados económicos de este proceso, las conclusiones de ingenieros y economistas no son todas iguales. Maldonado y Palma (2004), por ejemplo, enfatizan que los procesos de privatización del sector eléctrico en la región, especialmente en Chile, Colombia y Argentina, han estado caracterizados por un dinamismo de la inversión en el transcurso de los primeros años y un posterior decaimiento. Sin embargo, sostienen, el impulso inicial se debió más a la transferencia de activos existentes, que al aumento de la capacidad instalada de la red eléctrica.

Paredes-Molina (1995), por su parte, analiza el proceso de privatización chileno desde la intersección en- 
tre el sector eléctrico y el mercado de capitales, concluyendo que las reformas a este sector reforzaron y le dieron un impulso al mercado de capitales. Fisher y Serra (2007), por otro lado, consideran entre los beneficios de la privatización la caída de las tarifas para los clientes. No obstante, lo anterior es cierto solo para los clientes industriales (clientes libres), y no para los clientes regulados pues, tal como señalan Estache y Rodriguez-Pradina (2000), tras la privatización del sector eléctrico en Chile estos precios no cayeron.

En suma, si bien la privatización del sector eléctrico en Chile ha sido estudiada, los análisis generalmente se han hecho desde el punto de vista de la ingeniería y la economía. No obstante, recientemente se han producido aproximaciones sociohistóricas con la motivación de comprender el neoliberalismo desde este ámbito (Tironi y Barandiarán, 2014; Maillet y Rozas, 2017; Otaegui, 2017). A pesar de que el acercamiento de las ciencias sociales a la energía constituye un valioso avance hacia la comprensión del lugar que ocupó la energía en la sociedad en este periodo tan decisivo de la historia de Chile y de la región, hasta ahora no se ha dicho nada sobre las consecuencias que esta reorganización tuvo para los usuarios. Por ello, en este artículo se considera el marco teórico de los estudios de usuarios para analizar el caso del sector eléctrico en Chile.

\subsection{Estudios infraestructurales y de usuarios}

En la actualidad, y tal como plantean Collier, Mizes, y von Schnitzler (2016), el filósofo norteamericano John Dewey ha experimentado una renovada atención por los analistas de las infraestructuras. Especialmente influyente han sido sus observaciones sobre infraestructuras industriales, como aglutinadores de personas que, en la práctica, no han escogido estar juntas voluntariamente. En este sentido, para Collier y compañía (2016) las infraestructuras son «la fuerza más importante que da forma a los públicos» (Collier, Mizes, y von Schnitzler, 2016:5) que son vinculados por vastas corrientes de interconexión compleja. Así, las infraestructuras siempre presuponen un tipo de usuario en su diseño, de manera que el usuario está presente en todo el proceso de infraestructuración. Esto es lo que autores como Marres (2005) han destacado en sus análisis, pues, en el diseño de una infraestructura los usuarios son called into being (llamados a ser) de algún modo particular. En este sentido, una infraestructura es tanto una invitación como una constricción, y un llamado que invoca comportamientos y grupos que no son preexistentes (Barry, 2013).
Del mismo modo, las infraestructuras llaman a ser y se ensamblan en nombre de una promesa para sus usuarios, la cual, al margen de su cumplimiento efectivo, generalmente tiene una relevancia en tanto proposición sobre el futuro (Hetherington y Campbell, 2014), un relato que es tan contingente como histórico y que busca ser apoyado por la población (Appel, Anand y Gupta, 2018). En este sentido la infraestructuración puede ser vista como el resultado de un conglomerado de entidades que, al clasificar, y luego enrolar usuarios reproducen elecciones éticas, morales, prácticas, al mismo tiempo que articulan modos de convivencia nacional (Sánchez-Criado, 2016).

Clasificar y estandarizar por ende, es una práctica que se materializa, incrusta y automatiza en las infraestructuras (Bowker y Star, 2000) y que permite un tipo humano al mismo tiempo que constriñe otro. No obstante, no hay nada sencillo en este proceso: un usuario nunca se puede dar por garantizado (Sánchez-Criado, 2012). O como lo expresa Barry «sería un error imaginar que los públicos se forman de la manera que se espera o se pretende, o que los públicos son posibilidades preformadas que de alguna manera se crean o se ensamblan a través del uso de una técnica en particular» (2013: 192). Así, el usuario es producto de una cadena de acciones que va mucho más allá de una idea cerrada de humano. En esta dirección Sánchez-Criado define usuario como:

"disposiciones, arreglos en los que (i) las identidades y las capacidades no pueden ser atribuidas ni concentradas en torno a la figura de actores humanos preexistentes y encarnados; y que (ii) no derivan sólo del papel del lenguaje o de los puros determinantes tecnológicos, sino de entrelazamientos concretos de todos estos elementos en diferentes procesos» (Sánchez-Criado, 2012: 125).

Sánchez-Criado, además, se refiere a prácticas de apuntalamiento constantes como un factor imprescindible para mantener a un usuario, mediante movimientos rítmicos. Sin esa constancia, asegura este autor, el usuario podría dejar de existir fácilmente.

El usuario es capaz de forjar y resistir, encarnar o rechazan valores y códigos de ética y comportamientos prescritos por quienes diseñan los ensamblajes (Savage, 2018). Y, en efecto, la comprensión relacional destaca la agencia de los usuarios en asumir, ignorar, modificar o revertir las proposiciones inscritas en el diseño. Tal como lo han demostrado los estudios sobre usuarios, existe una variedad de antiprogramas 
(Akrich y Latour, 1992) mediante los cuales los usuarios rechazan o modifican las pautas preestablecidas. Así, mientras el programa es inscrito en los diversos dispositivos de las infraestructuras, permitiendo y prohibiendo a los usuarios un rango de acciones de manera anticipada, el antiprograma da cuenta de las acciones de los usuarios que entran en conflicto con lo prescrito por los programadores. Esta capacidad de los usuarios de modificar y apropiarse de los objetos que se usan, también ha sido señalada por las investigadoras feministas que acuñaron el concepto de domesticación (Oudshoorn, Saetnan, y Lie, 2002; van Oost, 2003). Este término ha sido empleado para nombrar la posibilidad de revertir el diseño en la apropiación y uso de los artefactos (Lie y Sørensen, 1996) y también como una manera de darle prominencia a la mirada del usuario sobre la del diseñador (Oudshoorn y Pinch, 2003).

De esta manera, y en consideración de la necesidad de profundizar en los conocimientos que se tienen de la relación infraestructura-usuario en el ámbito de la energía eléctrica, especialmente en el período de privatización que constituyó una nueva forma de administrar la energía y a sus públicos, esta investigación aborda las siguientes interrogantes: ¿de qué manera las políticas infraestructurales comprendieron y administraron a los usuarios en el período de privatización? ¿Qué papel jugó el modo en que estas políticas definieron, e inscribieron, a los usuarios en la infraestructura eléctrica? ¿Quiénes resistieron la versión de usuario proyectada desde las políticas infraestructurales?

\section{PERSPECTIVA GENEALÓGICA}

Cada vez son más las investigaciones que, desde los estudios de Ciencia, Tecnología y Sociedad (CTS) realizan análisis diacrónicos o genealógicos (Best, 1995; Flyvbjerg y Murillo, 2019), como una manera de hacer historia del presente (Foucault, 1977). De esta manera, hay quienes sugieren la necesidad de una ontología del movimiento (Nail, 2015), como una manera de visibilizar el dinamismo de propio de las entidades sociales. Quienes han aplicado esta mirada en el análisis de las políticas públicas (Savage y Lewis, 2018; Ureta, 2014; Youdell y McGimpsey, 2015) dan cuenta de la importancia de observar los componentes que se unen para un fin estratégico, como gobernar, pero también de su desintegración y resurgimiento en algo diferente. En efecto, si al mirar una política pública en el presente se podría obtener una ilusión de estabili- dad, al ampliar la mirada a un tiempo prolongado se desmontará (Savage y Lewis, 2018) y lo que se verá ya no es fijeza, sino cambio (Kingfisher, 2016; Wright y Shore, 2011; Tampio, 2009).

En el marco de la anterior perspectiva, para realizar la presente investigación se llevó a cabo el estudio detallado de un centenar de fuentes primarias correspondientes, principalmente, a la Administración Pública chilena. Los archivos seleccionados pertenecen al Archivo Nacional de la Administración (ARNAD), a la Comisión Nacional de Energía (CNE); a la Empresa Nacional de Electricidad (ENDESA); al Fondo Chilectra; a la Oficina de Planificación Nacional (ODEPLAN); al Ministerio de Minería, Ministerio de Economía, Fomento y Turismo; al Ministerio de Medio Ambiente; a la Biblioteca Nacional del Congreso; a la biblioteca de la Facultad de Filosofía y Humanidades de la Universidad de Chile; al Centro de Documentación del Ministerio de Vivienda y Urbanismo, y a la Hemeroteca de la Biblioteca Nacional.

Estos archivos contienen a su vez una amplia variedad de tipo de documentos: documentos de trabajo, comunicados de prensa, recortes de prensa, memorias, decretos, leyes, memorandos, informes de asesorías, correspondencia, discusiones parlamentarias, distintos tipos de asesorías; discursos presidenciales, estadísticas, conferencias, artículos académicos. Finalmente, se empleó el software Nvivo 11 para realizar un análisis cualitativo mediante Nodos. Los Nodos se estructuraron según dos niveles, uno temporal y otro conceptual (o de proceso). Asimismo, una serie de memos (herramienta Nvivo 11) fueron utilizados para complementar el análisis relacionando los modos de producción de los documentos, el tipo de conocimiento inscrito y de tecnologías empleadas. El análisis mediante Nvivo 11 hizo notorio uno de los casos más desafiantes para los expertos del periodo estudiado: los colgados. Por lo tanto, posteriormente, se realizó una reconstrucción cronológica a partir de la diversidad de archivos y tipos de fuentes que contenían referencias del caso para luego analizar en profundidad su contenido.

\section{EL CASO DE LOS COLGADOS}

\subsection{La privatización del sector eléctrico y el surgimiento del gobierno de los usuarios como antecedente}

En Chile, a partir de 1980 y luego de seis años de planificación, las infraestructuras del servicio público, como la electricidad, aguas, telecomunicaciones 
comenzaron a ser privatizadas y sus usuarios fueron organizados bajo nuevos criterios, categorías, clasificaciones y valores. En este ámbito la infraestructura eléctrica fue la pionera y una de sus empresas, Chilectra Metropolitana, fue empleada como experimento y más tarde como ejemplo para el resto de las infraestructuras básicas ${ }^{1}$. Así, mediante un método de ensayo-error se reorganizó toda esta industria. Si bien en este artículo no hay espacio para dar cuenta del proceso de privatización completo, cabe señalar tres movimientos relevantes.

En primer lugar, se trazó un modelo de privatización, testeado y consultado en reiteradas ocasiones con asesore ${ }^{2}$ e inversionistas tanto locales como internacionales ${ }^{3}$, que tendría como estrategia la fragmentación de las empresas públicas en múltiples filiales, divididas por rubro (generación, transmisión, y distribución) y por zonas de concesión. Con cada una de estas empresas se diseñaron «empresas de usuarios»: las compañías entregarían acciones, tanto a usuarios como trabajadores, como medio de pago cada vez que tuviesen que hacer devoluciones de dinero o pagar compensaciones (Centro de Estudios Públicos, 1992). Bajo esta perspectiva, se esperaba que la identificación por parte de los trabajadores y usuarios con las empresas, vendría asociada a la participación accionarial en las mismas.

En segundo lugar, se crearon instituciones de apariencia técnica que, a la vista de los usuarios, deberían funcionar de manera completamente independiente de los dueños del capital empresarial, así como de las motivaciones políticas. Así, se defendió la creación de una Comisión de carácter técnico, orientada a establecer metas, políticas energéticas nacionales, programas, presupuestos y fijar tarifas de cara al público ${ }^{4}$. Estas recomendaciones dieron paso a la creación de la Comisión Nacional de Energía (CNE) en 1978 como la institución técnica que regularía el sector. Por su parte los empresarios, que progresivamente se fueron convirtiendo en los nuevos dueños de las empresas eléctricas, quedaron relegados a una institución poco visible, como fue el Centro Económico de Despacho de Carga (CEDC).

En tercer lugar, se reorganizó a los usuarios de la electricidad: estos dejaron de ser pensados como una población homogénea, con tarifa única (Humeres, 2020), y comenzaron a administrarse dos categorías de clientes: los clientes libres y los clientes regulados. Con un consumo mayor de $250 \mathrm{kWh} /$ mes los clientes libres, principalmente clientes industriales, podrían negociar libremente su tarifa. Por otra parte, los clientes regulados, es decir, los usuarios residenciales quedarían sujetos a la regulación tarifaria de la CNE. Estas disposiciones, que comenzaron a ensayarse a partir de 1980, quedaron fijadas en la nueva Ley General de Servicios Eléctricos de $1982^{5}$. Estas clasificaciones de usuarios establecieron dos grandes zonas fundamentales para comprender la relación entre infraestructura y usuarios que se fundó en esta década y que perdura hasta la actualidad. Esta forma de clasificar y administrar usuarios daría origen a toda una política dedicada a gobernar usuarios. En este punto profundizaré a continuación.

\subsection{El gobierno de los usuarios: estrategias}

Las categorías diseñadas en el ámbito de la electricidad para contener y establecer relaciones específicas para cada grupo, regulados y libres, conllevaron una relación contractual y una tarifa que jamás volvería a ser estable y homogénea. Para los clientes regulados se configuró una nomenclatura compleja y variable. La fórmula constaría de indexadores dinámicos (en aquel momento alrededor de veinte); cálculos en base a una empresa modelo (empresa ficticia que, en teoría, sería la más eficiente de su área de concesión); y la tarifa se diferenciaría por área geográfica. Además, se creó la tarifa horaria y la tarifa de invierno ${ }^{6}$, bajo el supuesto de que cada usuario podía elegir no consumir electricidad en los horarios peak y en los meses de invierno (periodo compuesto por seis meses). Junto

1 Comisión Nacional de Energía, 1988. La experiencia chilena en el estudio e implementación de un sistema de tarificación eléctrica a costo marginal. Documento de Trabajo $N^{\circ} 13$. Fondo Comisión Nacional de Energía, Archivo Nacional de la Administración, Santiago, Chile. En adelante Fondo Comisión Nacional de Energía.

2 Oficina de Planificación Nacional, 1977. Estrategia Energética 1977-1978. Fondo ODEPLAN Archivo Nacional de la Administración, Santiago, Chile. En adelante Fondo ODEPLAN.

3 Comisión Nacional de Energía, 1982. Privatización del Sector Eléctrico. Factibilidad de interesar a inversionistas extranjeros. Documento de Trabajo N 33. Preparado por Claudio Valdés y Enrique Onetto. Fondo Comisión Nacional de Energía.

4 Oficina de Planificación Nacional, 1977. Estrategia Energética 1977-1978, p. 89. Fondo ODEPLAN.

5 Comisión Nacional de Energía, 1982. Ley General de Servicios Eléctricos en Chile. Documento de Trabajo N 52 . Fondo Comisión Nacional de Energía, Archivo Nacional de la Administración, Santiago, Chile. En adelante Fondo Comisión Nacional de Energía.

6 Comisión Nacional de Energía, 1980. Tarifas de distribución eléctrica. Documento de Trabajo º 68. Fondo Comisión Nacional de Energía. 
a la tarifa de invierno, además, se creó el «límite de invierno" fórmula que estableció un límite diferenciado según el consumo de verano de cada usuario, a partir del que se encarecería progresivamente su variación en los meses de invierno. De esta manera, la tarifa comenzó a ser pensada como un dispositivo de modelación de comportamiento y en una nomenclatura tremendamente variable e indescifrable para cada usuario.

Todos estos cambios, además de no producir un comportamiento informado-racional-económicamente por parte de los usuarios, produjeron un encarecimiento exponencial de la cuenta de la electricidad: comparando el mismo mes en 1982 y 1983, tal como lo reconocieron los expertos de la CNE «los usuarios experimentaron alzas en su boleta del invierno que podían llegar al $100 \%{ }^{7}$. Es decir, los horarios y meses peak solo produjeron un encarecimiento exponencial de la cuenta final. Dicho de otro modo, la opacidad y aparente automatismo de las fórmulas establecidas en este periodo, en efecto, programaron un aumento continuo de las tarifas in perpetuum.

\subsection{La emergencia del usuario rebelde: el caso Col- gados}

A pesar de que no es posible saber con precisión cuándo o en qué proporción los usuarios comenzaron a conectarse ilícitamente a la red eléctrica, nada parece indicar que fuese una práctica que emergiera en los ochenta. Así, la innovación de este momento estuvo en la creación de la categoría, en el estudio, y en la persecución de este tipo de usuarios. El caso de los colgados comenzó a ser una preocupación para el gobierno infraestructural a partir de 1980, momento en que los expertos comenzaron a destinar sus esfuerzos hacia la relación contractual, empresa-cliente. De este modo, los colgados concitaron bastante atención personal, de recursos y estrategias, convirtiéndose en un segmento fértil donde ensayar estrategias de gobierno y tácticas de control.

Según los registros de Chilectra Metropolitana, la empresa que en 1980 tenía el monopolio de la región, el caso de los colgados localizado en la Región Metropolitana alcanzó la cifra de $45.131^{8}$. Tras esta contabilización, Chilectra comenzó a enviar una boleta mensual con la cuenta de la electricidad a cada hogar identificándolo como colgado. Los dueños de Chilectra tenían muy claro que los colgados no tenían un medidor, por lo tanto, la boleta contenía un monto promedio que era figurado, por lo que aquellos números representaban una deuda imaginaria. Esta acción performativa buscaba concienciar a los colgados de que estaban siendo observados en su comportamiento fraudulento.

No obstante, los usuarios colgados continuaron aumentando. En 1982 se implementó el Plan Colgados ${ }^{9}$ que consistió en el empadronamiento de este tipo usuarios realizado por contingentes de carabineros y funcionarios municipales. Seguidamente, y como ninguna medida de registro y visibilización funcionaba por sí misma, se ordenó a los mismos carabineros y funcionarios municipales que arrancaran las conexiones ilícitas. Sin embargo, cuando la compañía dejaba de hacer los arranques de las conexiones ilegales, el número de colgados volvía a crecer.

Las medidas fueron escalando en envergadura y grados de amenaza. Se realizó una campaña en la prensa nacional mediante un comunicado que hacía un llamado a los usuarios a normalizar su situación:

«Por el perjuicio que su conducta encierra para esta Compañía y, en especial, para sus clientes, como asimismo por el peligro para la integridad física y bienes de terceros, CHILECTRA METROPOLITANA le notifica que perseguirá ante la Justicia del Crimen la aplicación de todo rigor de la Ley para quienes sean sorprendidos en la comisión del delito anteriormente señalado» ${ }^{10}$.

Al mismo tiempo, las iniciativas fueron avanzando hacia una estrategia punitiva: se comenzaron a enviar cartas a cada usuario colgado identificado, comunicándole que el delito del hurto de energía eléctrica conllevaba una pena que iba «desde 61 días a 5 años de presidio» ${ }^{11}$ según los grados de responsabilidad de cada cliente ilegal. Pero nada de esto consiguió el efecto esperado.

7 Comisión Nacional de Energía, 1983. Comentarios a La Minuta “Tarifas Eléctricas” de la SOFOFA. Documento de Trabajo N²9, p. 19. Fondo Comisión Nacional de Energía.

8 CHILECTRA, 1980. Programas Ministeriales 1980. Compañía Chilena de Electricidad S.A. Fondo Chilectra, Archivo Nacional de la Administración, p. 3. Santiago, Chile. En adelante Fondo Chilectra.

9 CHILECTRA, 1982. Deudas y consumidores en carpeta. Informes para señores alcaldes Región Metropolitana. Informe de Cesar Rodríguez Bravo, Director General Chilectra. Fondo Chilectra.

10 CHILECTRA, 1982, p. 13. Fondo Chilectra.

11 CHILECTRA, 1982, p. 11. Fondo Chilectra. 
En agosto de 1983 Chilectra creó una Oficina de Control de Pérdidas (OCP) para estudiar y monitorear constantemente a los colgados. Desde entonces esta Oficina tuvo como objetivo diseñar un Plan de Acciones de Control de Hurto ${ }^{12}$ y ejecutarlo midiendo los resultados periódicamente. Según el cálculo de la propia compañía, este comportamiento de los usuarios se traducía en una pérdida de hasta un $30 \%$ de la electricidad en los meses de invierno ${ }^{13}$. En 1986, el personal de la OCP vio superadas sus capacidades de control y contrató a la Universidad de Chile para realizar un estudio más detenido que analizara la situación completa y proporcionara recomendaciones.

Cuando el comportamiento de los colgados comenzó a ser materia de gobierno en 1980, este tipo de usuario había sido caracterizado como gente que vivía en campamentos, pobladores y también miembros de cooperativas rurales. A partir de entonces, se clasificó a los colgados en dos tipos: los usuarios colgados con y sin recursos económicos. De esta manera, el catastro de usuarios colgados comenzó a agruparlos según esta división, al mismo tiempo que se diseñaron planes diferenciados para enmarcar a los usuarios hacia el registro y pago de sus consumos. Por una parte, el Plan Zonal Masivo (PZM) fue diseñado para «inspeccionar permanentemente un Universo de alrededor de 600.000 usuarios de una condición socioeconómica superior ${ }^{14}$. Por otro lado, el Plan Atención Poblaciones (PAP) controlaba un grupo de 245.000 usuarios en poblaciones que, por lo general, hacían un arranque directo de la red. No obstante, a medida que Chilectra fue acumulando datos en el tiempo, especialmente tras la creación de un Departamento de Estadística dentro de la OCP, en 1986 los datos recogidos mostraban que los hurtos de electricidad eran tan significativos por parte de usuarios con recursos (PZM) como los sin recursos (PAP).

Tal como se señala en el documento Hurtos Sorprendidos entre 1983 y 1986 realizado por la Oficina de Control de Pérdidas, de Chilectra Metropolitanae en 1986, entre 1983 y 1984 los colgados con recur- sos eran significativamente más que los colgados sin recursos. En 1985 la cantidad era similar entre ambas categorías y solo durante 1986 los colgados sin recursos superaban considerablemente a los con recursos ${ }^{15}$. Por lo tanto, de estos datos se desprende que la cantidad de hurtos de energía eléctrica eran tan cuantiosos entre los usuarios con recursos como sin ellos. De este modo, se vio que los colgados no eran precisamente pobladores y campesinos como se había establecido desde Chilectra. Pero en vez de cuestionar si la categorización entre colgados con y sin recursos tenía algún sentido, desde la compañía se continuó describiendo el comportamiento de los usuarios y diseñando las políticas para gobernarlos, dentro del marco racional-económico. Tal como se ve a continuación, se estableció que si los 'colgados con recursos' hurtaban energía eléctrica sería por imitación (a los pobladores) o por vicio, inducidos por un tercero, o por costumbre:

«Residenciales: usuarios que tienen un ingreso familiar que les permite solventar los gastos de energía eléctrica y que hurtan por vicio inducidos por amistades o familiares, y por el ejemplo de tanto poblador "colgado" existente. Industriales y comerciales: que también tienen un nivel de ingreso adecuado y hurtan por los mismos motivos anteriores. Constructores: hurtan por costumbre...»16.

Así, las causas que motivaban a los usuarios con recursos, en todo momento fueron tratadas como un asunto marginal en relación a la razón de fondo que provocaba al surgimiento de un usuario colgado.

La organización del mundo en un esquema de coste-beneficio se traduciría en una imposibilidad, por parte del personal de la OCP, de comprender al usuario y sus propios datos más allá de la racionalidad económica. Como se puede apreciar en la siguiente cita, este esquema hacía que los analistas de usuarios interpretaran los resultados tal como ellos anticipaban: el motivo de todo colgado era económico, un comportamiento que en efecto respondía a la situación de

12 CHILECTRA, Oficina de Control de Pérdidas, 1986. Políticas y Estrategias Actuales Sobre Control de Hurtos de Energía Eléctrica Chilectra Metropolitana S.A. Estudio hecho por la Universidad de Chile. Fondo Chilectra.

13 CHILECTRA, Oficina de Control de Pérdidas, 1986, p. 10. Fondo Chilectra.

14 CHILECTRA, Oficina de Control de Pérdidas, 1986, p. 11. Fondo Chilectra.

15 1983: colgados con recursos 1.848; colgados sin recursos: 761.

1984: colgados con recursos: 20.420; colgados sin recursos: 18.592.

1985: colgados con recursos 10.273; colgados sin recursos: 11.157.

1986: colgados con recursos 5.717; colgados sin recursos: 11.095 .

Fuente: Hurtos sorprendidos entre 1983 y 1986. CHILECTRA, Oficina de Control de Pérdidas,1986. Fondo Chilectra.

16 CHILECTRA, Oficina de Control de Pérdidas, 1986, p. 2. Fondo Chilectra. 
la economía nacional. Así, tras el estudio realizado en 1986, los reportes de la OCP explicaron que:

«A pesar de la gran cantidad de antecedentes que generaron las dos encuestas realizadas sobre el problema a estudiar, se destaca y reafirma un resultado que de alguna manera se anticipaba. Una causa preponderante del hurto de energía son las secuelas de la recesión económica; un problema de disminución de ingresos. Las otras causas que se destacan a raíz del estudio son los usuarios que no son clientes (por ejemplo, sin medidor) y el hecho de estar moroso en el pago de las cuentas de electricidad»17.

Como se ve, pese a provocar alrededor de la mitad de los hurtos, los usuarios con recursos quedaron infra-representados. Al triangular toda la información, el reporte enviado a la CNE concluyó:

«Como se hizo ver al comentar el análisis estadístico de la información, la pobreza en primer lugar, la no condición de cliente de numerosos usuarios y la existencia de deuda morosa en el caso de otros, parecen ser los factores principales que determinan la ocurrencia del hurto, sin perjuicio de la presencia y el papel de la imitación social y el afán de ventaja. Pareciera, por tanto, que el hurto masivo en los sectores de extrema pobreza constituye en esencia un mecanismo por el cual estos sectores más desposeídos procuran aumentar su ingreso real»18.

Así, la llegada de los economistas al área de energía enmarcó todas las interpretaciones sobre el comportamiento de los usuarios en el modelo costo-beneficio.

La nueva estrategia que emprendió la OCP en alianza con los poderes estatales tuvo como único criterio la fe en un estímulo racional-económico para enmarcar a los usuarios rebeldes a una condición legítima: la fórmula que buscaban debía «incrementar el costo de incurrir en el hurto [e] incrementar los beneficios para clientes que cumplen con sus obligaciones»19. La deducción de los portavoces se expresaba de la siguiente manera:

«Pareciera, por lo tanto, que el hurto masivo en los sectores de extrema pobreza constituye en esencia un mecanismo por el cual estos sectores más despo- seídos procuran aumentar su ingreso real. Es por ello, que las medidas que surgen como recomendación, están orientadas a incrementar el costo de incurrir en hurto $y$, al mismo tiempo contemplan el reemplazo del ingreso real no captado por parte de quienes dejen de hurtar, por beneficios adicionales especialmente creados» 20.

En la cita anterior el marco interpretativo racional económico de los usuarios alcanza su máxima expresión. En este, la reducción del comportamiento de los colgados a la figura del usuario pobre, los lleva a inferir que el hurto es el resultado de un cálculo por aumentar el ingreso real. Además, como concluye la cita anterior, el personal de la OCP insta a la CNE a «reemplazar el ingreso real no captado» por beneficios para el cliente honesto, modelo opuesto del usuario colgado.

Para el grupo de usuarios sin recursos, la empresa solicitó que la CNE impulsara un programa de subsidios dirigido a los usuarios que no podían pagar el consumo mínimo. Este subsidio debía estar vinculado a otros beneficios "de la red social», y todos ellos debían estar condicionados por el buen comportamiento de los usuarios, para «incrementar las ventajas de ser buen cliente, dando los medios para ello y simultáneamente aumentado los costos por su no cumplimiento» 21 . En la carta, el condicionamiento del beneficio al buen comportamiento y su vinculación con la red de subsidios de protección social fue reiterado y destacado como la clave para el éxito del conjunto de medidas.

Al grupo marginal que formaban los usuarios con recursos, los que recordemos hurtaban la mitad del monto total de las pérdidas de electricidad en algunos períodos, se le intentó administrar «introduciendo algunas mejoras especialmente con miras a impedir la propagación por efecto demostración y afán de ventaja»22. Esto se tradujo en una estrategia diseñada para propiciar el autogobierno y así erradicar esas llamadas prácticas socialmente contagiosas. Para ello, se llevó a cabo una campaña global «de estímulo para el cliente honesto»23. Más adelante, la Campaña «Cliente Honesto» tuvo tácticas diferenciadas para cada tipo de usuario.

17 CHILECTRA, Oficina de Control de Pérdidas, 1986, p. 4. Fondo Chilectra.

18 CHILECTRA, Oficina de Control de Pérdidas, 1986, p. 5. Fondo Chilectra.

19 CHILECTRA, Oficina de Control de Pérdidas, 1986, p. 5. Fondo Chilectra.

20 CHILECTRA, Oficina de Control de Pérdidas, 1986, p. 6. Fondo Chilectra.

21 CHILECTRA, Oficina de Control de Pérdidas, 1986, p. 6. Fondo Chilectra.

22 CHILECTRA, Oficina de Control de Pérdidas, 1986, p. 7. Fondo Chilectra.

23 CHILECTRA, Oficina de Control de Pérdidas, 1986, p. 8. Fondo Chilectra. 


\section{CONCLUSIONES}

A partir del análisis anterior es posible destacar que la privatización del sector eléctrico en Chile tuvo consecuencias relevantes para la reorganización de la relación usuarios-infraestructura: si antes los usuarios/ clientes/consumidores de la energía eran pensados en términos generales, como una población homogénea cuyo vínculo se gestionaba mediante una tarifa única, con la privatización los usuarios fueron clasificados en subgrupos y reorganizados en términos contractuales, cuyo producto más visible fue una tarifa diferenciada. Asimismo, la racionalidad económica se convirtió en el principio más importante, sino el único, mediante el cual los usuarios comenzaron a ser interpretados y administrados por parte de los expertos. Este modelo racional-económico, comenzó a guiar el esquema tarifario de los usuarios regulados. Con ello se esperaba que la tarifa se convirtiera en un dispositivo de administración del comportamiento de los usuarios, con el cual se podría regular el consumo de energía según horarios, estaciones del año, o áreas geográficas, entre otras cosas.

Sin embargo, paradójicamente los expertos hicieron de la tarifa una nomenclatura indescifrable, opaca, que jamás fue comunicada de manera sencilla a los usuarios. De esta forma, el nuevo esquema resultó ser un mecanismo de incremento tarifario exponencial, que provoca que el precio final de la electricidad sea excesivamente caro, "dos veces los de la OCDE y tres los de Perú» (Rudnick y Palma, 2017: 21). Al mismo tiempo, la ininteligibilidad de la tarifa dificulta que la ciudadanía pueda discutir de estos temas que son fundamentales para la calidad de vida. No obstante, la interpretación unidimensional racional-económica de los usuarios por parte de los expertos quedó invalidada en su efecto, pero no en su uso. Los expertos continuaron aplicando esa interpretación y la consecuente administración de la relación usuario-infraestructura.

Por otra parte, además de las evidencias del fracaso de los supuestos que guiaron el esquema tarifario, tal como se observa en el caso de los colgados, la interpretación racional-económica parece obstaculizar cualquier tipo de evidencia que no se corresponda con dicho modelo. Como se ve en el análisis, aun cuando los estudios encargados por Chilectra arrojaban el resultado de que al menos la mitad de los usuarios colgados eran usuarios con recursos, los expertos continuaron explicando este tipo de comportamientos como un asunto de usuarios pobres que buscaban aumentar sus recursos económicos. De este modo, las estrategias y campañas para dirigir hacia la legalidad a este tipo de usuarios, tanto para los usuarios con, como sin recursos, se erigieron sobre estos supuestos de racionalidad económica, sin producir el éxito esperado. Es por este motivo que el hurto de energía eléctrica puede ser entendido como una resistencia, no necesariamente consciente, a la noción de usuario racional-económico proyectada por los expertos.

Por último, resulta pertinente recordar que el caso de los colgados fue reconstruido a partir de fuentes primarias, principalmente, archivos de la Administración Pública. Por lo tanto, el análisis se ha centrado en la perspectiva y valoraciones de los expertos, en cómo ellos definieron e intentaron administrar usuarios (colgados, honestos/deshonestos) según sus propios términos. Sin embargo, tal como han mostrado los estudios congregados en Palma (2019) sobre robo y justicia en América Latina, a partir de los hechos reconstituidos con fuentes primarias, también es posible advertir cómo el comportamiento de quienes infringen la ley desafía y cuestiona la legitimidad de las normas sociales, así como los métodos de persecución.

De cara al futuro, y más allá de Chile, el papel activo que los usuarios están tomado en la actualidad para ejercer sus demandas sobre las infraestructuras de servicios públicos, exige una mayor comprensión de la relación entre usuarios e infraestructuras por parte de las ciencias sociales. El fortalecimiento de los estudios infraestructurales en el último tiempo puede contribuir a la comprensión de otros casos en la región Latinoamericana. Después de todo, el caso chileno fue influyente para otros países no solo en términos de privatización del sector eléctrico (Maldonado y Palma, 2004, Bridge, Özkaynak y Turhan, 2018) sino también en cuanto a las reformas neoliberales (Plehwe, 2009; Dean, 2014) que produjeron una nueva administración de las infraestructuras (Hetherington y Campbell, 2014; Venkatesan, et al., 2018) y cambiaron la relación de estas entidades con la sociedad. Por lo tanto, futuros entrecruzamientos entre los estudios infraestructurales con foco en Latinoamérica con estudios de usuarios desde una perspectiva CTS (Collier, Mizes, y von Schnitzler, 2016; Bowker y Star, 2000; Sánchez-Criado, 2012; Akrich, 1992), podrían contribuir enormemente a pensar las infraestructuras de servicio público del siglo XXI, tanto en Chile como en otros países de la región. 


\section{AGRADECIMIENTOS}

Este artículo se realizó con el financiamiento del Núcleo Milenio de Investigación en Energía y Sociedad (NUMIES), ANID - Programa Iniciativa Científica
Milenio - Código NCS13_024. Asimismo, agradezco a al Dr. Sebastián Ureta, a las y los investigadores de NUMIES, por sus valiosos comentarios realizados a la investigación doctoral que dio origen a este artículo.

\section{REFERENCIAS}

Akrich, Madelaine (1992). The D-Scrption of Technical Objects. Shaping technology/building society. Cambridge MA: MIT Press.

Akrich, Madeleine; Callon, Michel; Latour, Bruno y Monaghan, Adrian (2002). The key to success in innovation part II: The art of choosing good spokespersons, International journal of innovation management, 6 (2): 207-225. https://doi. org/10.1142/S1363919602000562

Akrich, Madelaine y Latour, Bruno (1992). A summary of a convenient vocabulary for the semiotics of human and nonhuman assemblies. En: Webie Bijker y Jhon Law (eds.) Shaping Technology/ Building Society. Cambridge, MA: MIT Press.

Appel, Hannah; Anand, Nikhil; y Gupta, Akhil (2018). The Promise of Infrastructure. Durham: Duke University Press.

Arancibia, Patricia y Balart, Rodrigo (2007). Sergio de Castro: El arquitecto del modelo económico chileno. Editorial $\mathrm{Bi}$ blioteca Americana.

Barry, Andrew (2013). Material politics: Disputes along the pipeline. Oxford: John Wiley \& Sons. https://doi. org/10.1002/9781118529065

Best, Steven (1995). The Politics of Historical Vision Marx, Foucault, Habermas. Nueva York: Guilford. https://doi. org/10.4324/9780203985380

Bridge, Gavin; Özkaynak, Begüm y Turhan, Ethemcan (2018). Energy infrastructure and the fate of the nation: Introduction to special issue, Energy research \& social science, 4:1-11. https://doi. org/10.1016/j.erss.2018.04.029

Bowker, Geoffrey y Star, Susan (2000). Sorting things out: Classification and its consequences. Cambridge, MA: MIT Press. https://doi.org/10.7551/mitpress/6352.001.0001

Callon, Michel (1998). An essay on framing and overflowing: economic externalities revisited by sociology, The
Sociological Review, 46 (1_suppl): 244269. https://doi.org/10.1111/j.1467954X.1998.tb03477.x

Cass, Noel y Shove, Elizabeth (2018). Standards? Whose standards? Architectural Science Review, 61 (5): 272-279. https://doi.org/10.1080/00038628.20 18.1502158

Castán-Broto, Vanesa (2017). Energy landscapes and urban trajectories towards sustainability. Energy Policy 108 (2017): 755-764. https://doi.org/10.1016/j. enpol.2017.01.009

Cavallo, Ascanio; Sepúlveda, Oscar, y Salazar, Manuel (1989). La Historia Oculta del Régimen Militar: Chile 1973-1988. Santiago: Editorial Antártica.

Centro de Estudios Públicos (1992). El Ladrillo: bases de la política económica del gobierno militar chileno. Santiago, Chile: Centro de Estudios Públicos.

Collier, Stephen; Mizes, James y Von Schnitzler, Antina (2016). Public Infrastructure/Infrastructural Publics. Limn, (7). Disponible en: https://limn.it/ issues/public-infrastructuresinfrastructural-publics/ [julio 2016; 12 de mayo 2020].

Dean, Mitchell (2014). Rethinking neoliberalism, Journal of Sociology, 50 (2): 150-163. https://doi. org/10.1177/1440783312442256

Dewey, John (1991). The Public and Its Problems. Athens, $\mathrm{OH}$ : Swallow Press/Ohio University Press.

Estache, Antonio y Martimort, David (2000). Transaction costs, politics, regulatory institutions and regulatory outcomes. En: Luigui Manzetti (ed.) Regulatory Policy in Latin America: Post-Privatization Realities, North-South Press Center at the University of Miami, pp. 49-82

Graham, Steve y Marvin, Simon (2001). Splintering Urbanism: Networked Infrastructures, Technological Mobilities, and the Urban Condition. London, New York: Routledge. https://doi. org/10.4324/9780203452202
Farías, Ignacio (2011). Ensamblajes urbanos: la TAR y el examen de la ciudad. Athenea Digital. Revista de pensamiento e investigación social 11 (1): 15-40. https://doi.org/10.5565/rev/athenead/v11n1.826

Fischer, Karin (2009). The Influence of Neoliberals in Chile before, during, and after Pinochet. En Plillip Mirowski y Dieter Plehwe (eds.) The road from Mont Pèlerin: The making of the neoliberal thought collective, with a new preface. Harvard University Press. https://doi. org/10.4159/9780674054264-010

Fischer, Ronald y Serra, Pablo (2007). Efectos de la privatización de servicios públicos en Chile. Washington DC: Banco Interamericano de Desarrollo.

Flyvbjerg, Bent, y Murillo, Lorena (2019). Habermas y Foucault: ¿pensadores de la sociedad civil? Estudios Sociológicos, 19 (56): 295-324.

Foucault, Michel (1977). Language, Counter-Memory, Practice. Ithaca, NY: Cornell University Press.

Ffrench-Davis, Ricardo (2003). Entre el neoliberalismo y el crecimiento con equidad. Tres décadas de política económica en Chile. 3a ed. Santiago: J. C. Sáenz.

Gárate, Manuel (2012). La revolución capitalista de Chile (1973-2003). Santiago: Ediciones Universidad Alberto Hurtado.

Harvey, Penny y Knox, Hannah (2015). Roads: An anthropology of infrastructure and expertise. Ithaca, NY: Cornell University Press.

Hetherington, Kregg y Campbell, Jeremy (2014). Nature, infrastructure, and the state: Rethinking development in Latin America, The Journal of Latin American and Caribbean Anthropology, 19 (2): 191-194. https://doi.org/10.1111/ jlca.12095

Humeres, Mónica (2020) Usuario modelo: un análisis sociotécnico del lugar de los usuarios en las políticas de la infraestructura eléctrica en Chile (1935-2019) [Tesis Doctoral inédita]. Universidad Alberto Hurtado, Santiago. 
Kaltenbrunner, Annina y Painceira, Juan Pablo (2018). Subordinated financial integration and financialisation in emerging capitalist economies: the Brazilian experience. New political economy, 23(3): 290-313. https://doi.org/10.1080/13563 467.2017.1349089

Kingfisher, Catherine (2016). A policy travelogue: Tracing Welfare reform in Aotearoa/New Zealand and Canada. Nueva York: Berghahn.

Klein, Naomi, y Fuentes, Isabel (2007). La doctrina del shock: el auge del capitalismo del desastre (Vol. 1). Barcelona: Paidós.

Kreimer, Pablo (2006). ¿'Dependientes o integrados? La ciencia latinoamericana y la nueva división internacional del trabajo. Nómadas, 24: 199-212.

Lüders, Rolf. 21 de diciembre de 2012. Roberto Kelly y los Chicago Boys, La Tercera.

Maldonado, Pedro y Palma, Rodrigo (2004). Seguridad y calidad del abastecimiento eléctrico a más de 10 años de la reforma de la industria eléctrica en países de América del Sur. Santiago: CEPAL.

Maillet, Antoine y Rozas, Joaquín (2017). Hybridization of neoliberalism policies: The case of the Chilean electric power policy reform (2014-2016). Gestión y política pública, 28 (1): 207235. https://doi.org/10.29265/gypp. v28i1.546

Marres, Noortje (2005). Issues Spark a Public into Being: A Key but Often Forgotten Point of the Lippmann Dewey Debate. En: Bruno Latour y Peter Weibe (eds.). Making Things Public. Cambridge, MA: MIT Press, pp.208-217.

Nail, Thomas (2015) The figure of the migrant. Stanford: Stanford University Press.

Otaegui, Álvaro (2017). Formateando el mercado [eléctrico] en Chile. Genealogía del proceso de (des)enmarcación energética en tiempos del neoliberalismo (1976-1978), Núcleo Milenio de Investigación en Energía y Sociedad. Disponible en: https://numies.cl/publicaciones [actualizada el 19 de agosto del 2018; citada el 09 de septiembre 2021].

Oudshoorn, Nelly; Rudinow, Ann, y Lie, Merete (2002). On gender and things: Reflections on an exhibition on gendered artifacts, Women's Studies International Forum, 25 (4): 471-483. https://doi. org/10.1016/S0277-5395(02)00284-4

Oudshoorn, Nelly y Pinch, Trevor (2003). How users matter. The co-construction of users and technology. Cambridge, Massachusetts: MIT Press. https://doi. org/10.7551/mitpress/3592.001.0001

Palma, Daniel (2019). Ladrones, policías y orden callejero en Santiago de Chile, 18961924. Anuario Colombiano de Historia Social y de la Cultura, 46(2): 59-86. https:// doi.org/10.15446/achsc.v46n2.78214

Paredes-Molina, Ricado (1995). El sector eléctrico y el mercado de capitales en Chile. CEPAL.

Pirez, Pedro (2013). La urbanización y la política de los servicios urbanos en América Latina. Andamios, (22): 4567. https://doi.org/10.29092/uacm. v10i22.266

Plehwe, Dieter (2009). Introduction. En: Philip Mirowski y Dieter Plehwe (eds.) The Road from Mont Pèlerin. The Making of the Neoliberal Thought Collective. Cambridge: Harvard University Press, pp. 1-34. https://doi. org/10.4159/9780674054264-001

Rudnick, Hugh y Palma, Rodrigo (2017). Contexto nacional e internacional en marzo de 2014. En: Pacheco, Máximo (ed.) Revolución energética en Chile. Santiago de Chile: Ediciones UDP, pp.19-41.

Sánchez-Criado, Tomas (2012) ¿Cómo se mantiene una usuaria? Prácticas de apuntalamiento en la teleasistencia para personas mayores. En: Francisco Tirado y López, Daniel (eds.) Teoría del Actor-Red: Más allá de los estudios de ciencia y tecnología. Barcelona: Amentia Editorial, pp. 111-156.

Sánchez-Criado, Tomas (2016). Pensar infraestructuralmente. INMATERIAL. Diseño, Arte y Sociedad, 1 (1): 85-98. https://doi.org/10.46516/inmaterial. v1.17

Savage, Glenn (2019). What is policy assemblage? Territory, Politics, Governance, 8(3): 319-335. https://doi.org/1 $0.1080 / 21622671.2018 .1559760$

Savage, Glenn y Lewis, Steven (2018). The phantom national? Assembling national teaching standards in Australia's federal system, Journal of Education
Policy, 33 (1): 118-142. https://doi.org /10.1080/02680939.2017.1325518

Star, Susan (1999). The ethnography of infrastructure, American behavioral scientist, 43 (3): 377-391. https://doi. org/10.1177/00027649921955326

Star, Susan y Ruhleder, Karen (1995). Steps toward an ecology of infrastructure: Design and access for large information spaces, Information systems research, 7 (1): 111-134. https://doi. org/10.1287/isre.7.1.111

Star, Susan y Bowker, Geoffrey (2006). How to infrastructure. En Leah Lievrouw y Sonia Livingstone. Handbook of new media: Social shaping and social consequences of ICTS. London: SAGE Publications, pp. 151-162. https://doi. org/10.4135/9781848608245.n12

Tampio, Nicholas (2009). Assemblages and the Multitude: Deleuze, Hardt, Negri, and the Postmodern Left, European Journal of Political Theory, 8 (3): 383-400. https://doi. org/10.1177/1474885109103850

Tironi, Manuel y Barandiarán, Javiera (2014). Neoliberalism as Political Technology: Expertise, Energy, and Democracy in Chile. En Eden Medina, Ivan Costa Marques, y Christina Holmes, Beyond imported magic: essays on science, technology, and society in Latin America, Boston: MIT Press, pp. 305-329. https://doi.org/10.7551/ mitpress/9780262027458.003.0015

Undurraga, Tomás (2014). Divergencias: trayectorias del neoliberalismo en Argentina y Chile. Santiago: Ediciones Universidad Diego Portales.

Ureta, Sebastián (2014). Policy assemblages: proposing an alternative conceptual framework to study public action, Policy studies, 35 (3): 303-318. https://doi.org/ 10.1080/01442872.2013.875150

Valdés, Juan-Gabriel (1995). Pinochet's Economists: The Chicago School in Chile. Cambridge, Inglaterra, Nueva York: Cambridge University Press.

Van Oost, Elizabeth (2003). Materialized gender: how shavers configure the users' feminity and masculinity. En Nelly Oudshoorn y Trevor Pinch (eds.) How users matter. The co-construction of users and technology. Cambridge, Massachusetts: MIT Press, pp.193-208. 
Varsavsky, Oscar (1969). Ciencia, política, cientificismo, Buenos Aires: Centro Editor de América Latina.

Velho, Raquel y Ureta, Sebastián (2019). Frail modernities: Latin American infrastructures between repair and ruination, Tapuya: Latin American Science, Technology and Society, 2 (1): 1-14. https://doi. org/10.1080/25729861.2019.1678920

Venkatesan, Soumhya; Bear, Laura; Harvey, Penny; Lazar, Sian; Rival, Laura y AbdouMaliq, Simone (2018). Atten- tion to infrastructure offers a welcome reconfiguration of anthropological approaches to the political, Critique of Anthropology, 38 (1): 3-52. https://doi. org/10.1177/0308275X16683023

Wright, Susan y Shore, Cris (2011). Conceptualising policy: Technologies of governance and the politics of Visibility. En: Cris Shore, Susan Wright y Davide Peró (eds.) Policy worlds: Anthropology and the analysis of contemporary power. Nueva York: Berghahn, pp. 1-26.
Wilkie, Alex y Michael, Mike (2009). Expectation and mobilisation enacting future users, Science, Technology y Human Values, 34 (4): 502-522. https://doi. org/10.1177/0162243908329188

Youdell, Deborah y McGimpsey, Ian (2015). Assembling, disassembling and reassembling 'youth services' in Austerity Britain, Critical Studies in Education, 56 (1): 116-130. https://doi.org/10.1080/ 17508487.2015 .975734 